\title{
Correspondence
}

\section{The Cassel Hospital}

\section{DeAR Sirs}

We wish to both commend and amend the paper by Drs Bowen \& Bremner (Psychiatric Bulletin, September 1990, 14, 542-543) on their experience as rotating registrars at the Cassel Hospital. In their balanced and considered account, they thoroughly recommend the training experience available at the Cassel in the in-patient units where disturbed patients are treated as individuals and as family members. Assessment of suitability for psychotherapy and treatments such as brief focal therapy, marital therapy, family therapy, and group therapy under supervision in the out-patients department add to the "rich academic and clinical milieu".

Alas, since acceptance of this article for publication, one rotating registrar post has been cut at the Cassel by Riverside Health Authority, thus drastically reducing the option for a whole-time psychotherapy placement to Riverside trainees. Riverside's loss may be a gain to other districts who, guided by clinical and training needs, may wish to fund or part-fund a registrar training post at the Cassel.

Finally, it is important to clarify the current position of the Cassel Hospital in relation to the changing NHS. The editorial note at the foot of the paper is factually incorrect and misleading. The District Health Authority's closure proposal was withdrawn following agreement on plans to raise funds to offset a cut of $£ 300,000$ in the hospital's revenue for this financial year. This represents a massive $25 \%$ cut in our budget, and with our backs to the wall we are prepared to do all we can to ensure the continued survival of this "internationally renowned clinical and training centre". We are not opting out of the NHS, and wish to continue to provide an essential life-changing service to families and individuals in need.

The Cassel Hospital

DAVID BELL

KeVIN Healy

Richmond, Surrey TW10 7JF

See pp. 7-8.

\section{Writing to the patient}

\section{DeAR SIRS}

Drs Price \& Asch (Psychiatric Bulletin, August 1990, 14, 467-469) have drawn attention to the benefits of writing to the patient. I do not recall this topic being discussed in any British journals and I think that the authors have opened an important topic for discussion. In some areas of Australian and New Zealand psychiatry there has for several years been pioneering work in letter writing. Price \& Asch come to the conclusion that only good can come of writing letters to patients. David Epston \& Michael White, two antipodean psychotherapists, have taken this approach further and have proposed that letters can be important therapeutic aids. It would be an exaggeration to say that letter writing is widespread in psychiatric practice in Australia and New Zealand, but it is becoming increasingly used as a technique.

I would commend to anyone who wishes to explore the subject to make Epston \& White's book Literate Means to Therapeutic Ends (1989) their starting point. As well as a theoretical background, the authors present their thinking behind a dozen different types of letter, and include a wealth of illustrative case material. The book can be obtained from Dulwich Centre Publications, c/o Kensington Consultation Centre, 47 South Lambeth Street, London SW8 1RH.

South Shields General Hospital

JIM BIRCH

South Shields, Tyne \& Wear NE34 OPL

\section{The white coat syndrome}

\section{DeAR SIRS}

I read with interest Dr Wear's article on the 'White Coat of the Liaison Psychiatrist' (Psychiatric Bulletin, September 1990, 14, 540-541).

In conducting a research study in the surgical service, I found that in our first week we had a completion rate of our questionnaires of less than $30 \%$. However, in looking at out practice we decided that this might be due to the fact that we were not perceived as part of the surgical team. Therefore, my colleague and I donned white coats and I am happy to report that our completion rate increased to approximately $95 \%$.

Peter L. Jenkins

St Cadoc's Hospital

Caerleon, Gwent NP6 1XQ

\section{Beyond mental hospital sites}

DEAR Sirs

I read with interest the paper by Tannahill et al (Psychiatric Bulletin, July 1990, 14, 399-401). While 
agreeing with the general thrust of the paper, I must challenge their statement that provision for all patients with special needs for continuing care and challenging behaviours could be provided away from mental hospital sites.

As a forensic psychiatrist, I see chronically psychotic patients who pose intractable management problems such as violence or inappropriate sexual behaviour. These are referred to the forensic service because they cannot be safely managed within an acute DGH unit or a continuing care hostel in the community. Unfortunately, the needs of these patients are not well met by regional secure units, with their emphasis on shorter term, medium security care. A recent survey of patients in Broadmoor Hospital from the South Western Region (Smith et $a l$, in preparation) indicated that a significant number no longer required treatment in conditions of maximum security. However, in the absence of local facilities catering for chronically disturbed psychotic patients, they remained inappropriately in a special hospital.

There may only be very few patients requiring long-term medium security in each district. However, these patients' needs are considerable and should not be overlooked. If one accepts that there is a need for both a degree of security and a comprehensive range of back-up facilities to ensure a reasonable quality of life, it is unrealistic to assume that community units isolated from other services can provide these.

I find it hard to envisage anywhere but a hospital campus, albeit much reduced in size and redesigned, possibly organised on a sub-regional or regional basis, being able to provide the necessary care for this group of patients.

JEANETTE SMITH

Devon and Cornwall Forensic Psychiatry Service Butler Clinic, Langdon Hospital

Dawlish EX7 ONR

\section{References}

Smith, J. E., Gordon, H. \& Donovan, W. M. Patients in Broadmoor Hospital from the South Western Region. (In preparation.)

Tannahill, M., Wilkinson, G. \& Higson, P. (1990) Beyond mental hospital sites. Psychiatric Bulletin, 14, 399-401.

\section{DeAR Sirs}

We too are concerned about the currently inadequate residential and rehabilitative provision for people with special psychiatric needs in both continuing care and challenging behaviour contexts. Action rather than rhetoric is required, and there remains great scope for the introduction and application of diverse and imaginative solutions to the many problems facing affected individuals, their carers, health authorities, social services departments, and policy makers. In the event, some authorities may well establish provision on mental hospital sites. In doing so they will have accepted the well known pitfalls of such a policy, in particular the professional isolation inherent in small remote sites, the danger of institutionalisation, and worse, the potential for even greater neglect of this group in a descending spiral of low morale, high staff turnover and inevitably declining standards of clinical care in the absence of quality control and peer review. Such is the stuff of Enquiries.

The central issue is that the work of caring for people with intractable severe mental illness appears to be an unattractive occupation, personally demanding, lacking in status, and unrewarding financially and as a career. The practical result is a major problem in recruiting professionals of all disciplines to provide the appropriate mix of skills and high quality treatment for this group.

It is imperative that steps are taken to seek to ensure the attraction, recruitment and training of professionals from all disciplines to work with people with severe and chronic mental illnesses as this is likely to determine the quality of services provided. Management systems must be appropriately supportive of such staff and staff-patient ratios ought to be weighted not only by physical dependency but also by psychological dependency. Career planning, regular staff appraisal, and financial incentives may be required to avoid rapid turnover of staff and 'burnout'. It should be a requirement of clinical training that some time be spent in the care of people with chronic mental illness. Efforts should also be made to encourage clinical research in the area of chronic mental illness as this serves to develop critical awareness of the related problems as well as serving as a stimulus to improve standards and encourage innovation.

The quality of the care provided to people with chronic mental illness is a crucial measure of the adequacy of mental health service provision. By this yardstick present provision is failing.

Greg WILKINSON

M. M. TANNAHILL PETER Higson

Clwyd Health Authority

North Wales Hospital

Denbigh LL16 5SS

\section{Audit of admissions to a psychiatric intensive care unit}

\section{Dear Sirs}

We would like to describe our findings from two surveys of admissions into a psychiatric intensive care unit at the North Wales Hospital. Opened in 